\title{
Differential roles for NOD2 in osteoblast inflammatory immune responses to bacterial pathogens of bone tissue
}

\author{
Vinita S. Chauhan and lan Marriott \\ Department of Biology, University of North Carolina at Charlotte, Charlotte, NC 28223, USA
}

Correspondence

lan Marriott

imarriot@uncc.edu

\begin{abstract}
Osteoblasts produce an array of immune molecules following bacterial challenge that can contribute to inflammation and the recruitment of leukocytes to sites of infection during bone diseases such as osteomyelitis. However, the mechanisms by which osteoblasts perceive and respond to facultative intracellular pathogens such as Salmonella species and Staphylococcus aureus have not been determined. Recently, our laboratory has described the expression in osteoblasts of members of the nucleotide-binding domain leucine-rich repeat region containing family of proteins that include nucleotide-binding oligomerization domain-2 (NOD2), a molecule that functions as an intracellular receptor for bacterial peptidoglycans. In the present study, we demonstrate that NOD2 expression is required for select inflammatory mediator production by osteoblasts following infection with the invasive pathogen Salmonella. In contrast, we have found that the inflammatory immune responses of osteoblasts to the passively internalized bacterial species Staphylococcus aureus, heat-killed pathogenic Salmonella, a non-invasive Salmonella strain and specific Toll-like receptor ligands are not reduced in the absence of NOD2 expression but are, in fact, elevated. Based upon these findings, we suggest that NOD2 serves differential roles in osteoblasts, promoting inflammatory responses to invasive bacteria while tempering cell responses to extracellular and/or passively internalized bacterial species.
\end{abstract}

Received 7 September 2009

Accepted 26 March 2010

\section{INTRODUCTION}

Studies from our laboratory have demonstrated that osteoblasts exposed to Salmonella enterica and Staphylococcus aureus, the principle causative agents of osteomyelitis, express an array of soluble and cell surface molecules that could promote immune responses and osteoclast activity at sites of bone infection (as summarized by Marriott, 2004 and Marriott et al., 2007). The demonstration that osteoblasts express members of the Toll-like family of cell surface pattern recognition receptors (TLR) may represent a means by which these cells respond to such pathogens (Kikuchi et al., 2001; Gasper et al., 2002; Madrazo et al., 2003). However, our previous studies have shown that bacterial products, inactivated bacteria and invasion-defective strains are far less potent than viable pathogenic bacteria at eliciting these responses (Bost et al., 1999, 2000, 2001; Gasper et al., 2002). These results suggest that bacterial invasion is required for maximal osteoblast responses and point to the involvement of additional intracellular sensors for bacterial pathogens or their products.

Abbreviations: GFP, green fluorescent protein; MDP, muramyl dipeptide; NLR, nucleotide-binding domain leucine-rich repeat region; NOD2, nucleotide-binding oligomerization domain-2; TLR, Toll-like receptor.
Consistent with this hypothesis, we have recently demonstrated that osteoblasts express nucleotide-binding oligomerization domain-2 (NOD2) (Marriott et al., 2005), a member of the novel nucleotide-binding domain leucinerich repeat region containing family of proteins (NLR) that functions as an intracellular receptor for bacterial peptidoglycans (Girardin et al., 2003; Inohara et al., 2003). The functional nature of such expression was inferred from the ability of TLR ligands or bacterial challenge to upregulate the expression of both NOD2 and Rip2 kinase (also designated RICK and CARDIAK) (Marriott et al., 2005), a critical downstream effector molecule in the NOD2 signalling pathway (Chin et al., 2002; Kobayashi et al., 2002). Furthermore, we demonstrated that muramyl dipeptide (MDP), a ligand for NOD2 (Girardin et al., 2003; Inohara et al., 2003), significantly augments TLRinduced inflammatory cytokine production by osteoblasts (Marriott et al., 2005). It is therefore possible that NOD2 acts as a sensor for facultative intracellular bacterial species such as Salmonella and Staphylococcus aureus in this cell type.

In the present study, we have assessed the role played by NOD2 in the inflammatory responses of primary osteoblasts to invasive and extracellular and/or passively internalized bacterial species. We report that NOD2 
expression contributes to inflammatory cytokine production by Salmonella-infected osteoblasts. In contrast, osteoblast responses to Staphylococcus aureus are augmented in the absence of NOD2 expression and resemble the enhanced responses observed with inactivated Salmonella, a non-invasive Salmonella strain, and ligands for cell surface TLRs.

\section{METHODS}

Reagents and solutions. LPS isolated from Escherichia coli (>500000 EU mg ${ }^{-1}$; $\geqslant 97 \%$ by TLC) and the MDP MurNAc-LAla-D-isoGln ( $\geqslant 98 \%$ by TLC) were purchased from Sigma Chemical Company. An activating oligonucleotide, GCTCATGACGTTCCTGATGCTG, previously described as a specific agonist for TLR9 (Sweet et al., 2002), was synthesized by Integrated DNA Technologies. Endotoxin-free flagellin protein preparations were isolated from Salmonella typhimurium serotype 12023 as previously employed (Madrazo et al., 2003). Doses selected represent those that were empirically determined to elicit optimal responses.

Wild-type and NOD2-deficient mice. B6.129S1-Nod2 $2^{t m 1 F l v} / \mathrm{J}$ mice that are NOD2-deficient (NOD2-/-) and bred for $>10$ generations onto a C57BL/6 background, as well as wild-type C57BL/6j mice, were purchased from Jackson Laboratory.

Isolation and characterization of murine osteoblasts. Primary osteoblast cell cultures were prepared from C57BL/6 and NOD2deficient mouse neonate calvariae by sequential collagenaseprotease digestion as previously described by our laboratory (Bost et al., 1999; Marriott et al., 2005; McCall et al., 2008). Osteoblasts isolated in this manner have previously been characterized as being $>99 \%$ pure cultures as determined by their distinctive morphology, and by the expression of type I collagen, osteocalcin, alkaline phosphatase and parathyroid hormone-cAMP (Bost et al., 1999).

Exposure of cultured osteoblasts to Salmonella enterica or Staphylococcus aureus. Osteoblasts were exposed to bacteria as previously described by our laboratory (Bost et al., 1999; Marriott et al., 2005; McCall et al., 2008). Confluent cell layers of cells were exposed to the pathogenic Salmonella enterica serovar Typhimurium strain SB300, Salmonella enterica serovar Typhimurium strain SB136 or Staphylococcus aureus UAMS-1 (ATCC 49230) at the indicated ratios of bacteria to osteoblasts in growth medium without antibiotics for $45 \mathrm{~min}$ at $37^{\circ} \mathrm{C}$. Following the infection period, cell cultures were washed with PBS and incubated in growth medium with $25 \mu \mathrm{g}$ gentamicin $\mathrm{ml}^{-1}$ to kill remaining extracellular bacteria. Salmonella enterica serovar Typhimurium strain SB136 is an invasion-defective mutant strain of Salmonella enterica serovar Typhimurium strain SB300, due to a mutation in the invA gene that prevents Salmonella pathogenicity island-1 type III secretion mechanisms. Staphylococcus aureus UAMS-1 is a clinical isolate and capable of inducing inflammatory responses in osteoblasts. In some experiments, osteoblasts were exposed to green fluorescent protein (GFP)expressing Staphylococcus aureus (Staphylococcus aureus RN4220 $\mathrm{p}^{\mathrm{SB} 2030}$ ) (Reott et al., 2008). SB136 and GFP-expressing Staphylococcus aureus were kind gifts from Dr Michael C. Hudson at The University of North Carolina at Charlotte.

Quantification of inflammatory cytokine and chemokine secretion in bone protein extracts and osteoblast culture supernatants. Specific capture ELISAs were performed to quantify IL-6, IL-12p70, MCP-1, IP-10 and RANKL levels in culture supernatants as described previously by our laboratory (Bost et al., 1999; Marriott et al., 2005; McCall et al., 2008).

Immunoblot analysis of NOD2 and GRIM-19. Immunoblot analyses for the presence of NOD2 and GRIM-19 in osteoblasts were performed as described previously by our laboratory (Gasper et al., 2002; Marriott et al., 2005; McCall et al., 2008). The primary antibodies used were a rabbit serum antibody directed against NOD2 (IMGENEX) and a mouse antibody that is reactive against murine GRIM-19 (BD Transduction Laboratories). To assess total protein loading in each well, immunoblots were reprobed with a goat anti-mouse $\beta$-actin antibody (Santa Cruz Biotechnology).

Co-immunoprecipitation of Rip2 kinase with NOD2. Coimmunoprecipitation was performed as described by our laboratory (McCall et al., 2008). Protein A agarose beads (Pierce Endogen) were pre-incubated with antibodies directed against NOD2 for $1 \mathrm{~h}$ prior to incubation with cell lysates for $18 \mathrm{~h}$ at $4{ }^{\circ} \mathrm{C}$. The immunoprecipitated material was subsequently subjected to immunoblot analysis for the presence of Rip2 kinase (Assay Designs). Since most standard housekeeping proteins are lost during this two-step process, we have determined the level of expression of an unidentified non-specific protein (irrelevant protein) that elutes in the immunoprecipitated material to confirm equal loading in each lane.

Measurement of bacterial burden and apoptotic volume decreases by flow cytometry. Bacterial burden in osteoblasts challenged with GFP-expressing Staphylococcus aureus was assessed by flow cytometry (FACSCalibur; Becton Dickinson) as previously described by our laboratory (McCall et al., 2008). In some experiments, proportions of cells undergoing apoptosis were determined by measuring apoptotic volume decreases as changes in forward and side scatter characteristics by flow cytometry as previously described by our laboratory (McCall et al., 2008).

Densitometric analyses. Densitometric analyses of immunoblots were performed using NIH Image (obtained from the NIH website: http://rsb.info.nih.gov/nih-image). Results are presented as arbitrary densitometric units corrected for background intensity and normalized to the expression of $\beta$-actin, or as fold increases over levels in unstimulated cells.

Statistical analysis. All results are presented as the mean \pm SEM and were tested statistically by Student's $t$-test and one- or two-way analysis of variance (ANOVA) with Tukey's post-hoc test, as appropriate, using commercially available SAS v9.1.3 software. Results were considered to be statistically significant at a probability of $<0.05$.

\section{RESULTS AND DISCUSSION}

\section{NOD2 expression is required for maximal expression of select inflammatory mediators by primary osteoblasts following in vitro Salmonella infection}

Osteomyelitis is a refractory bone disease and can be recurrent with attacks occurring following treatment even when causative organisms can no longer be isolated from sites of infection (as discussed by Marriott et al., 2007). An explanation for these phenomena may lie in the ability of the causative agents of osteomyelitis to invade and persist within resident bone cells. Internalization may provide a 
means of protection against neutrophil and antibodymediated immune responses, and may complicate treatment of bone infections by limiting exposure to antibiotics. Salmonella is a widely studied invasive bacterial pathogen, and we have previously demonstrated the ability of this bacterium to invade and persist within osteoblasts (Bost et al., 2001). Such a finding is not surprising since Salmonella is well known for its ability to invade nonleukocytic cells (Ohl \& Miller, 2001). The recent description of the NLR family of cytosolic proteins that can function as pattern recognition receptors for bacterial motifs has provided a potential mechanism by which both immune and non-immune cell types can perceive intracellular bacterial pathogens (as reviewed by Ting \& Davis, 2005; Strober et al., 2006). We have previously described the expression of several NLR family members, including CIITA and NLRP3, in osteoblasts (Schrum et al., 2003; McCall et al., 2008). Importantly, we have also demonstrated that these cells can express NOD2 (Marriott et al., 2005), a NLR that can recognize a minimal bacterial peptidoglycan motif that is found in both Gram-positive and Gram-negative organisms (Girardin et al., 2003; Inohara et al., 2003).

While distinct NOD2-mediated responses to intracellular bacteria have been described in immune cells including macrophages (Kobayashi et al., 2005; Leber et al., 2008), in the present study we have performed experiments using isolated primary cultures of osteoblasts derived from NOD2 $+1+$ and NOD2 $-1-$ animals to determine whether this intracellular pattern recognition receptor similarly mediates inflammatory immune responses of these resident bone cells to Salmonella. As shown in Fig. 1(a), NOD2-/- derived osteoblasts produce significantly lower levels of IL-6 at $12 \mathrm{~h}$ following Salmonella infection than cells from $\mathrm{NOD} 2+/+$ animals. Interestingly, our data suggest the presence of redundant mechanisms underlying perception of Salmonella by osteoblasts as the differences in the maximal production of IL- 6 in the absence or the presence of NOD2 could be overcome when cells were exposed to high bacterial numbers (Fig. 1a). The importance of NOD2 expression in Salmonella-induced osteoblast immune responses was not restricted to IL-6 as the production of a second key inflammatory cytokine, IL-12p70, was similarly lower in the absence of NOD2 at $24 \mathrm{~h}$ post-infection (Fig. 1b). However, NOD2 does not appear to be important for all osteoblast immune responses to this invasive bacterial pathogen as evidenced by the absence of an effect of NOD2 deficiency on the production of the inflammatory chemokine MCP-1 at $12 \mathrm{~h}$ (data not shown) or $24 \mathrm{~h}$ (Fig. 1c) post-infection.

Yang et al. (2005) have previously suggested that NOD2mediated signalling can exacerbate TLR-induced increases in the level of mRNA encoding the osteoclastogenic factor RANKL in osteoblasts. We have therefore determined whether NOD2 deficiency affects RANKL production by osteoblasts following Salmonella challenge. In the present
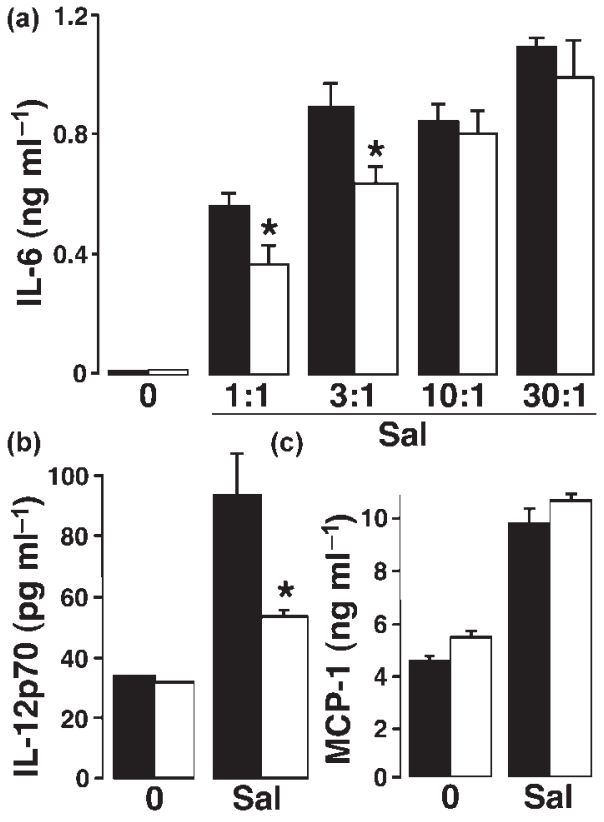

Fig. 1. NOD2 expression is required for maximal expression of select inflammatory mediators by primary osteoblasts following in vitro Salmonella infection. Cells $\left(2 \times 10^{6}\right)$ derived from wild-type (NOD2 $+/+$; black bars) or NOD2-deficient (NOD2-/-; white bars) mice were untreated (0) or exposed to Salmonella (1:1, $3: 1,10: 1$ and $30: 1$ bacteria to each osteoblast). At $12(\mathrm{a} ; n=7)$ or 24 (b, c; m.o.i. of $10: 1 ; n=3) \mathrm{h}$ post-infection, culture supernatants were isolated and levels of IL-6, IL-12p70 and MCP-1 were assessed by specific capture ELISA. Asterisks indicate significant differences between similarly treated NOD2 + 1 + and NOD2-/- derived cells $(P<0.05)$.

study, we have found no significant difference in bacterially induced RANKL secretion in the presence or absence of NOD2 expression $\left(31.5 \pm 3.3 \mathrm{pg} \mathrm{ml}^{-1}\right.$ in $\mathrm{NOD} 2+/+$ derived cells versus $32.2 \pm 0.1 \mathrm{pg} \mathrm{ml}^{-1}$ in NOD2-/derived cells following Salmonella challenge at an m.o.i. of $10: 1 ; n=4)$. Taken together, these data indicate that NOD2 plays a significant role in the detection of an invasive pathogen by osteoblasts and the subsequent production of select cytokines that could promote protective immune responses or progressive inflammatory damage.

\section{Production of select inflammatory mediators by osteoblasts following exposure to Staphylococcus aureus is elevated in the absence of NOD2}

We have compared our findings obtained with this invasive bacterial species with those obtained with a second pathogen of bone tissue, Staphylococcus aureus, an organism that is passively internalized by osteoblasts (Ellington et al., 1999). As shown in Fig. 2(a), in vitro exposure to Staphylococcus aureus is a potent stimulus for 

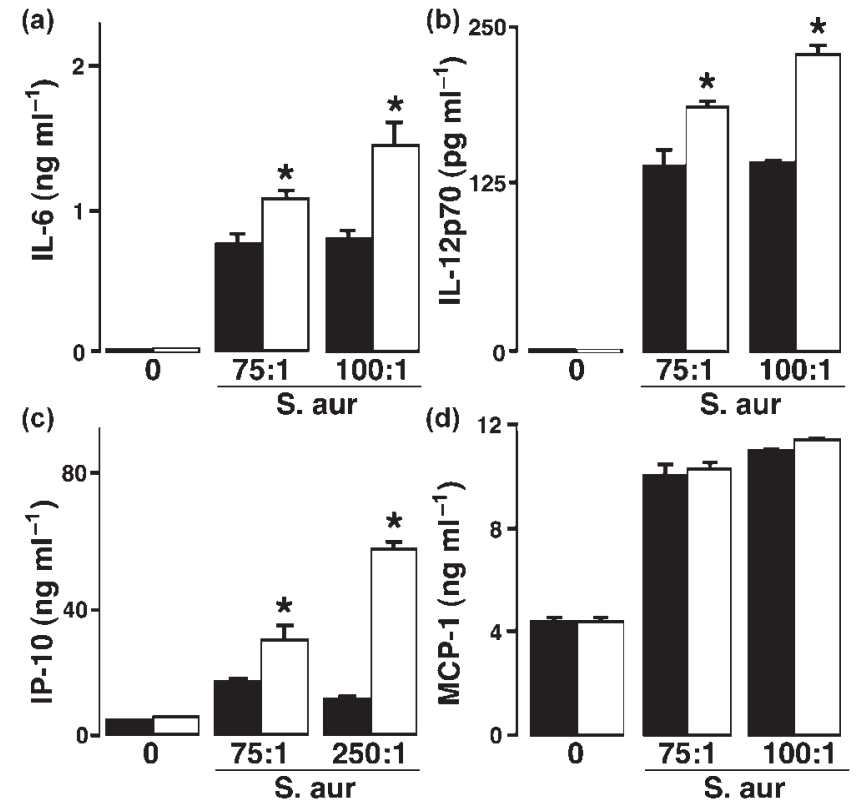

Fig. 2. Expression of select inflammatory mediators by osteoblasts following in vitro exposure to Staphylococcus aureus is elevated in the absence of NOD2. Cells $\left(2 \times 10^{6}\right)$ derived from wild-type (NOD2 +/+; black bars) or NOD2-deficient (NOD2-/-; white bars) mice were untreated (0) or exposed to Staphylococcus aureus (75:1, $100: 1$ and $250: 1$ bacteria to each osteoblast). At $12(\mathrm{a})$ or $24(\mathrm{~b}-\mathrm{d}) \mathrm{h}$ post-challenge, culture supernatants were isolated and levels of IL-6, IL-12p70, IP-10 and MCP-1 were assessed by specific capture ELISA. Asterisks indicate significant differences between similarly treated NOD2 +/ + and NOD2-/derived cells $(n=3 ; P<0.05)$.

IL-6 production by osteoblasts at $12 \mathrm{~h}$ following challenge. Interestingly, and in contrast to the responses to Salmonella, osteoblast production of IL- 6 is elevated in cells isolated from NOD2 $-/-$ mice relative to wild-typederived cells. Again, this effect was not restricted to IL-6 and Staphylococcus aureus-induced production of IL-12p70 (Fig. 2b) and IP-10 (Fig. 2c) was similarly higher in NOD2-/- cell cultures at $24 \mathrm{~h}$ following bacterial challenge. Consistent with our findings with Salmonella, the influence of this NLR appears to be selective as there were no significant differences in the production of MCP-1 by these cells in the absence or presence of NOD2 expression at $12 \mathrm{~h}$ (data not shown) or $24 \mathrm{~h}$ (Fig. 2d) following bacterial challenge, or in the level of production of RANKL $\left(34.3+1.7 \mathrm{pg} \mathrm{ml}^{-1}\right.$ in NOD2 $+/+$ derived cells versus $32.6 \pm 1.1 \mathrm{pg} \mathrm{ml}^{-1}$ in NOD2-/- derived cells following Staphylococcus aureus challenge at an m.o.i. of $75: 1 ; n=4)$.

It is conceivable that the absence of NOD2 leads to a higher bacterial burden within Staphylococcus aureus-infected osteoblasts thereby providing a more potent stimulus for inflammatory mediator production. To investigate this possibility, we have assessed the bacterial burden of
NOD2 $+/+$ and NOD2 $-/-$ derived cell cultures following challenge with GFP-expressing Staphylococcus aureus. As shown in Fig. 3, NOD2 $-/-$ derived cells demonstrated a modest but statistically significant $(P<0.05 ; n=5)$ increase in the proportion of osteoblasts harbouring bacteria at $24 \mathrm{~h}$ following in vitro challenge than similarly challenged wild-type cells. Another possibility is that the presence or absence of NOD2 could influence osteoblast survival following infection. However, no differences in the proportion of cells undergoing apoptotic volume decreases were observed between bacterially challenged NOD2 + / + and NOD2-l- cells as determined by flow cytometric analysis (data not shown). Perhaps more importantly, differences in bacterial burden or changes in lethality fail to explain why the production of certain inflammatory mediators such as IL-6, and IL-12p70 and IP-10 is either promoted or decreased in the presence or absence of NOD2 expression, while the production of other soluble proteins including MCP-1 and RANKL is unaltered.

\section{Inflammatory cytokine production by osteoblasts exposed to non-invasive Salmonella is elevated in the absence of NOD2}

Alternatively, the apparent differences in NOD2-mediated cell responses to Salmonella and Staphylococcus aureus could stem from the dissimilar mechanisms by which these organisms are internalized by osteoblasts. While both of these organisms are facultative intracellular pathogens of osteoblasts, Salmonella utilizes a type III secretion system to actively invade host cells (Ohl \& Miller, 2001), while the

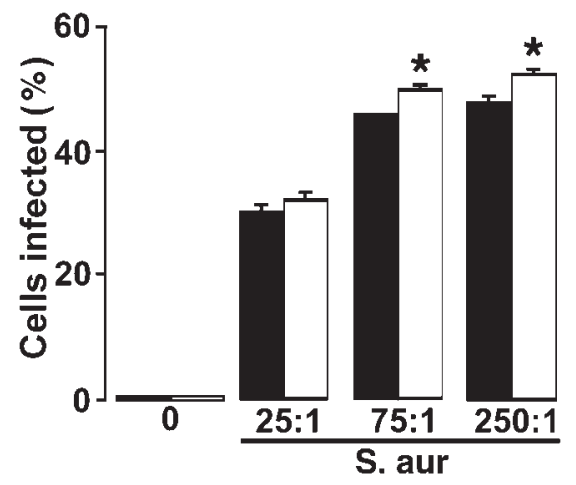

Fig. 3. Osteoblast cultures derived from NOD2-deficient animals demonstrate a somewhat higher bacterial burden following exposure to Staphylococcus aureus than similarly challenged wild-type derived cells. Cells $\left(2 \times 10^{6}\right)$ derived from wild-type (NOD2 +/+; black bars) or NOD2-deficient (NOD2-/-; white bars) mice were untreated (0) or exposed to GFP-expressing Staphylococcus aureus (25:1, $75: 1$ and $250: 1$ bacteria to each osteoblast). At $24 \mathrm{~h}$ post-treatment, cells were analysed for the proportion of cells demonstrating GFP-associated fluorescence as determined by flow cytometry. Asterisks indicate significant differences between similarly treated NOD2 +/ + and NOD2-/derived cells $(n=5 ; P<0.05)$. 
entry of Staphylococcus aureus into osteoblasts is passive (Ellington et al., 1999). To test this hypothesis, we examined cell responses to heat-killed pathogenic Salmonella or a non-invasive Salmonella strain in the absence or presence of NOD2 expression. As shown in Fig. 4, and consistent with our previous studies (Bost et al., 1999; Marriott et al., 2005), IL-6 production by osteoblasts
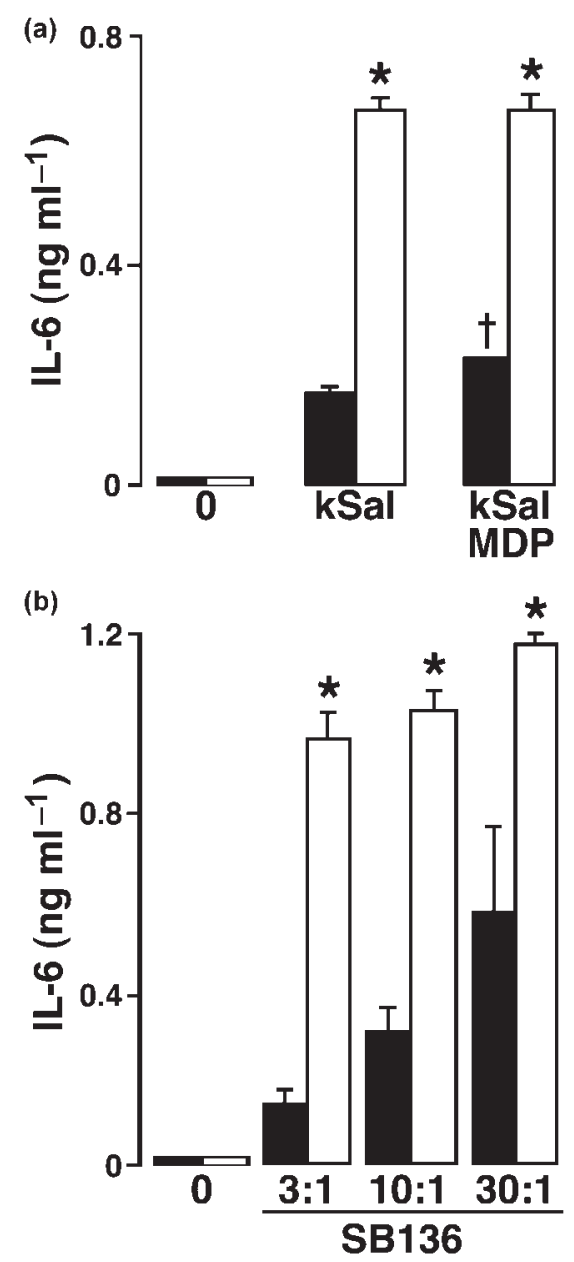

Fig. 4. Expression of $\mathrm{IL}-6$ by osteoblasts following in vitro exposure to heat-killed Salmonella or a non-invasive Salmonella strain (SB136) is elevated in the absence of NOD2. (a) Cells $\left(2 \times 10^{6}\right)$ derived from wild-type (NOD2 $+/+$; black bars) or NOD2-deficient (NOD2-/-; white bars) mice were untreated (0) or exposed to heat-killed Salmonella (30:1 bacteria to each osteoblast) in the presence or absence of MDP $\left(5 \mu \mathrm{g} \mathrm{ml}^{-1}\right)$ for 24 h. (b) Cells $\left(2 \times 10^{6}\right)$ derived from NOD $2+/+$ (black bars) or NOD2-/- mice (white bars) were untreated (0) or exposed to SB136 (3:1, 10:1 and 30:1 bacteria to each osteoblast) for $12 \mathrm{~h}$. Levels of IL-6 in culture supernatants were assessed by specific capture ELISA. Asterisks indicate significant differences between similarly treated NOD2 $+/+$ and NOD2-/- derived cells while the dagger symbol indicates a significant difference between groups in the absence or presence of MDP $(n=3$; $P<0.05)$. exposed to either killed invasive Salmonella (Fig. 4a) or SB136, an invasion-deficient strain of Salmonella (Fig. 4b), is markedly lower than that seen following infection with an invasive strain (Fig. 2a). Interestingly, and in contrast to IL-6 levels following infection with viable invasive Salmonella, osteoblast production of IL-6 is elevated in cells derived from NOD2-1- mice following exposure to killed Salmonella (Fig. 4a) or SB136 (Fig. 4b) relative to wild-type derived cells in a similar manner to that seen following Staphylococcus aureus challenge. Taken together, these data suggest that NOD2 differentially influences osteoblast immune responses to actively invasive versus passively internalized bacteria.

\section{Inflammatory cytokine production by osteoblasts exposed to select TLR ligands is elevated in the absence of NOD2}

These findings suggest that NOD2 mediates osteoblast immune responses to actively invasive bacterial pathogens, but attenuates responses to external/passively internalized organisms. To further test this hypothesis, we have investigated cell responses to specific ligands for various TLRs in the absence or presence of NOD2 expression. As shown in Fig. 5, exposure of osteoblasts to LPS, flagellin or an activating oligonucleotide (CpG), which are ligands for TLR4, TLR5 and TLR9, respectively, induces the expression of IL-6, while MDP alone fails to induce inflammatory cytokine production by osteoblasts (data not shown) as previously reported by our laboratory (Marriott et al., 2005). Similar to our results with Staphylococcus aureus and non-invasive Salmonella, IL-6 production by LPS- or flagellin-challenged osteoblasts was higher in cells derived from NOD2-/- mice than wild-type-derived osteoblasts (Fig. 5a, b). Interestingly, this phenomenon appears to be restricted to cell surface TLR types as IL-6 responses to a specific ligand for TLR9, an endosomal pattern recognition receptor, were not significantly different between cells derived from NOD2 $+/+$ and NOD2 $-/-$ animals (Fig. 5c).

Consistent with this finding, we have confirmed that the NOD2 ligand, MDP, can augment the expression of IL-6 elicited by either LPS or flagellin but not CpG, and it is important to note that MDP-mediated augmentation of LPS and flagellin-induced IL-6 production is not apparent in similarly treated cells derived from NOD2 $-/-$ mice (Fig. 5). These findings are consistent with the studies of Netea et al. (2005) demonstrating that MDP can modulate TLR2- and TLR4-mediated cytokine responses in human mononuclear cells, but not those initiated by TLR9. Taken together, these data suggest that NOD2 can limit cell surface TLR-mediated inflammatory cytokine responses in osteoblasts, although it remains to be determined whether such an effect represents a constitutive activity of NOD2 in its unbound state or reflects the actions of an as yet undetermined endogenous ligand. 

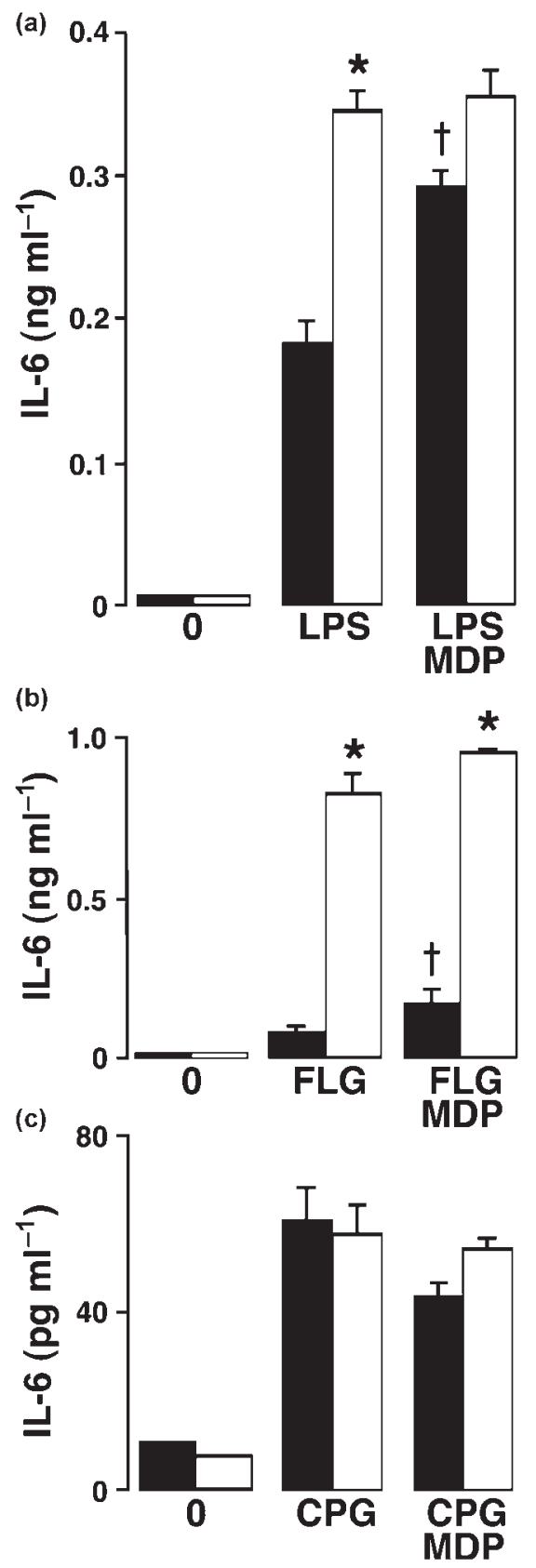

Fig. 5. Expression of $\mathrm{IL}-6$ by osteoblasts following in vitro exposure to select TLR ligands is elevated in the absence of NOD2. Cells $\left(2 \times 10^{6}\right)$ derived from wild-type (NOD2 $+/+$; black bars) or NOD2-deficient (NOD2-/-; white bars) mice were untreated (0) or exposed to LPS (100 $\mathrm{ng} \mathrm{m}^{-1}$ ), flagellin (FLG; $100 \mathrm{ng} \mathrm{ml}^{-1}$ ) or an activating oligonucleotide (CpG; $\left.1 \mu \mathrm{g} \mathrm{ml}^{-1}\right)$ in the absence or presence of MDP (5 $\mathrm{g} \mathrm{ml}^{-1}$ ). At 12 (a) or 24 (b, c) h post-challenge, culture supernatants were isolated and levels of IL-6 were assessed by specific capture ELISA. Asterisks indicate significant differences between similarly treated NOD2 + / + and NOD2-/- derived cells while the dagger symbol indicates a significant difference between groups in the absence or presence of MDP $(n=3 ; P<0.05)$.

\section{Salmonella, but not Staphylococcus aureus, elicits activation of a NOD2-associated downstream effector molecule}

Finally, we have investigated the relative ability of Salmonella and Staphylococcus aureus to elicit activation of NOD2-associated downstream effector molecules. Rip2 kinase is a critical downstream effector molecule in the NOD2 signalling pathway in other cell types (Chin et al., 2002; Kobayashi et al., 2002). As shown in the representative immunoblot of three separate experiments in Fig. 6(a), we have used co-immunoprecipitation techniques to demonstrate that infection of osteoblasts with Salmonella elicits association of NOD2 with Rip2 kinase. Salmonella-infected osteoblasts show an almost eightfold induction at an m.o.i. of 10:1 bacteria to each osteoblast over the low level of association observed in uninfected cells. In contrast, exposure of osteoblasts to Staphylococcus aureus fails to induce appreciable NOD2/Rip2 association (Fig. 6a) despite being able to elicit a significant increase in NOD2 protein expression (Fig. 6b).

While Rip2 kinase activation has been widely shown to be a critical step in the initiation of cellular responses following NOD2 ligation, at least one report has suggested that NOD2 can associate with a second adaptor/effector molecule, GRIM-19 (gene associated with retinoid-interferon-induced mortality-19) (Barnich et al., 2005). To determine whether this molecule has the potential to play a role in NOD2-mediated osteoblast responses to bacterial challenge, we have investigated the expression of GRIM-19 in this cell type at rest and following Salmonella or Staphylococcus aureus infection. As shown in the representative immunoblot of three separate experiments in Fig. 6(c), osteoblasts fail to express detectable levels of this adaptor molecule constitutively. Importantly, while GRIM19 protein expression was induced in cells at $24 \mathrm{~h}$ following Staphylococcus aureus or Salmonella exposure, osteoblasts failed to express detectable levels of this molecule up to $2 \mathrm{~h}$ following bacterial challenge (Fig. 6c). As such, it appears unlikely that GRIM-19 plays a significant role in rapid NOD2-mediated osteoblast cell responses to bacterial pathogens.

Together, these findings suggest that the inflammatory immune responses of osteoblasts to invasive bacterial pathogens such as Salmonella, but not passively internalized bacterial species including Staphylococcus aureus, are mediated at least in part via NOD2 activation of Rip2 kinase.

\section{Conclusions}

The present study supports and extends our earlier description of constitutive NOD2 expression in osteoblasts and the ability of bacterial pathogens and/or their components to upregulate such expression (Marriott et al., 2005). Furthermore, the present findings are consistent with the recent work of other investigators describing the 

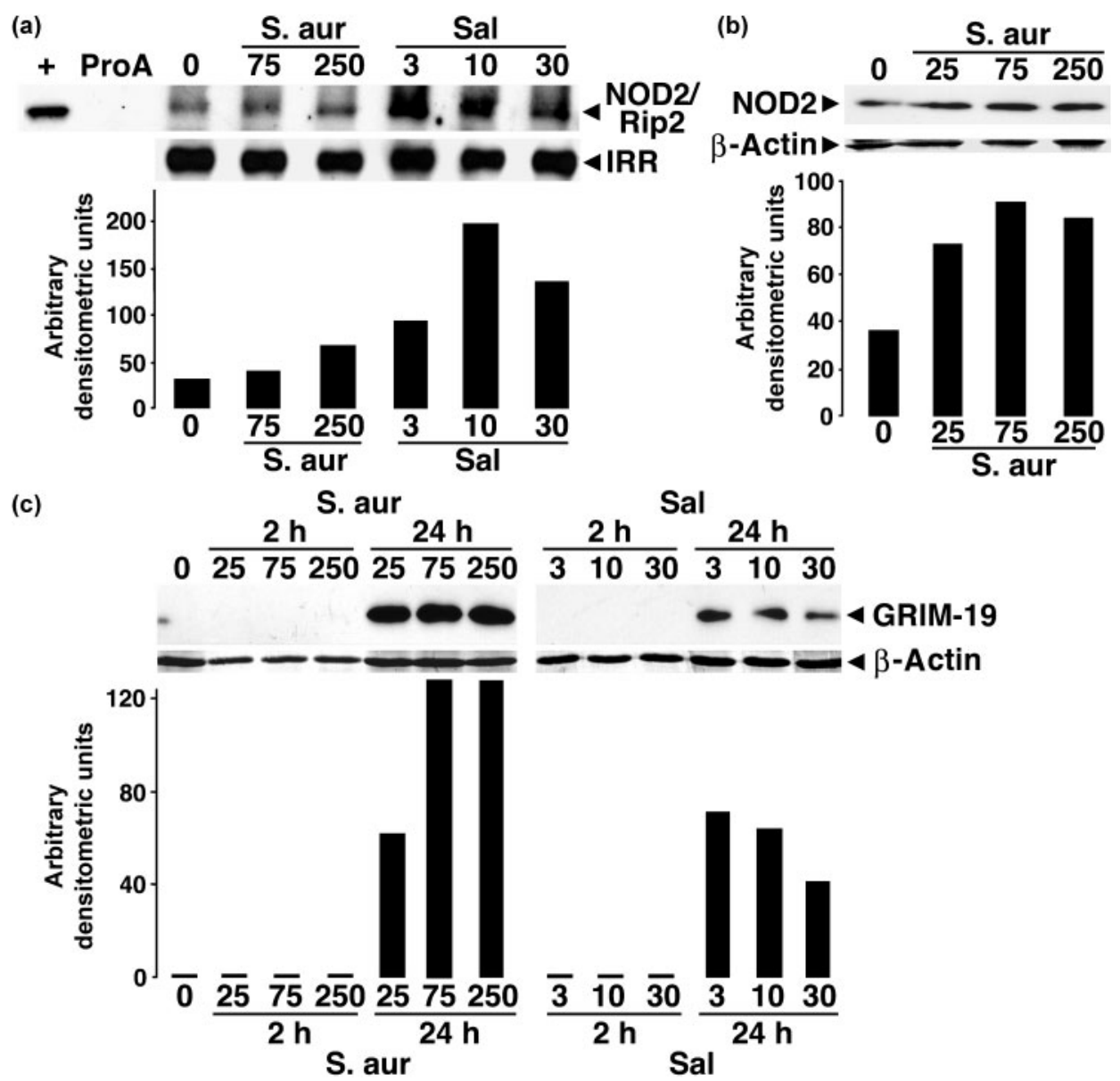

Fig. 6. Salmonella elicits more robust activation of NOD2-associated downstream effector molecules in osteoblasts than Staphylococcus aureus. (a) Osteoblasts $\left(2 \times 10^{6}\right.$ per well) were untreated (0) or exposed to Staphylococcus aureus (75: 1 and $250: 1$ bacteria to each osteoblast) or Salmonella (3:1, 10:1 and $30: 1$ bacteria to each osteoblast). After 2 h, protein isolates were immunoprecipitated with antibodies directed against NOD2 and subsequently analysed by denaturing immunoblot for the presence of Rip2 kinase. As a negative control, samples exposed to the immunoprecipitation procedure in the absence of antibodies against Rip2 kinase and run on the same immunoblots are shown (ProA). As a positive control, samples from thymus tissue were subjected to the same procedure and run on the same immunoblot $(+)$. To confirm equal loading in each lane, the expression of an irrelevant protein present in the immunoprecipitated material is shown (IRR). (b) Cells $\left(2 \times 10^{6}\right.$ per well) were untreated (0) or exposed to Staphylococcus aureus (25:1, 75:1 and 250:1 bacteria to each osteoblast) for $12 \mathrm{~h}$ prior to whole-cell protein isolation and immunoblot analysis for NOD2 expression. (c) Cells $\left(2 \times 10^{6}\right.$ per well) were untreated (0) or exposed to Staphylococcus aureus (25:1, $75: 1$ and 250:1 bacteria to each osteoblast) or Salmonella (3:1, $10: 1$ and $30: 1$ bacteria to each osteoblast) for 2 or $24 \mathrm{~h}$ prior to protein isolation and immunoblot analysis for GRIM-19 expression. Bar graphs showing densitometric analysis of each representative immunoblot from three separate experiments normalized to the expression of an irrelevant protein (IRR) or $\beta$-actin are shown.

expression of NOD2 in primary murine osteoblasts (Yang et al., 2005), and osteoblast and cementoblast-like cell lines (Nemoto et al., 2008). We have previously demonstrated that a NOD2 ligand can augment the production of IL-6 by stimulated osteoblasts (Marriott et al., 2005) and similar results have been obtained in cementoblasts (Nemoto et al., 2008). The data presented in this study support the functional expression of this NLR in primary osteoblasts and suggest that NOD2 plays differential roles in the regulation of immune responses by this cell type. As might be predicted, this intracellular pattern recognition receptor appears to play an important role in the generation of osteoblast inflammatory responses to an invasive bacterial species. However, NOD2 also appears to play an alternative function in osteoblasts, namely the suppression of inflammatory mediator production by extracellular and/or passively internalized bacterial pathogens. At the present time, the reasons for this unexpected second function are unclear but it is tempting to speculate that such suppression might represent a protective host mechanism to limit infectionassociated inflammatory damage in bone tissue. The idea that NLRs can also suppress innate immune responses is 
supported by the recent demonstration that NOD1 deficiency elevates acute inflammation in a murine model of arthritis (Joosten et al., 2008). While further study will be required to resolve this issue, it is apparent that NOD2 plays a significant role in the control of the innate immune functions of osteoblasts.

\section{REFERENCES}

Barnich, N., Hisamatsu, T., Aguirre, J. E., Xavier, R., Reinecker, H. C. \& Podolsky, D. K. (2005). GRIM-19 interacts with nucleotide oligomerization domain 2 and serves as downstream effector of anti-bacterial function in intestinal epithelial cells. J Biol Chem 280, 19021-19026.

Bost, K. L., Ramp, W. K., Nicholson, N. C., Bento, J. L., Marriott, I. \& Hudson, M. C. (1999). Staphylococcus aureus infection of mouse or human osteoblasts induces high levels of interleukin-6 and interleukin-12 production. J Infect Dis 180, 1912-1920.

Bost, K. L., Bento, J. L., Ellington, J. K., Marriott, I. \& Hudson, M. C. (2000). Induction of colony-stimulating factor expression following Staphylococcus or Salmonella interaction with mouse or human osteoblasts. Infect Immun 68, 5075-5083.

Bost, K. L., Bento, J. L., Petty, C. C., Schrum, L. W., Hudson, M. C. \& Marriott, I. (2001). Monocyte chemoattractant protein-1 expression by osteoblasts following infection with Staphylococcus aureus or Salmonella. J Interferon Cytokine Res 21, 297-304.

Chin, A. I., Dempsey, P. W., Bruhn, K., Miller, J. F., Xu, Y. \& Cheng, G. (2002). Involvement of receptor-interacting protein 2 in innate and adaptive immune responses. Nature 416, 190-194.

Ellington, J. K., Reilly, S. S., Ramp, W. K., Smeltzer, M. S., Kellam, J. F. \& Hudson, M. C. (1999). Mechanisms of Staphylococcus aureus invasion of cultured osteoblasts. Microb Pathog 26, 317-323.

Gasper, N. A., Petty, C. C., Schrum, L. W., Marriott, I. \& Bost, K. L. (2002). Bacterium-induced CXCL10 secretion by osteoblasts can be mediated in part through toll-like receptor 4. Infect Immun 70, 40754082.

Girardin, S. E., Boneca, I. G., Viala, J., Chamaillard, M., Labigne, A., Thomas, G., Philpott, D. J. \& Sansonetti, P. J. (2003). Nod2 is a general sensor of peptidoglycan through muramyl dipeptide (MDP) detection. J Biol Chem 278, 8869-8872.

Inohara, N., Ogura, Y., Fontalba, A., Gutierrez, O., Pons, F., Crespo, J., Fukase, K., Inamura, S., Kusumoto, S. \& other authors (2003). Host recognition of bacterial muramyl dipeptide mediated through NOD2. Implications for Crohn's disease. J Biol Chem 278, 5509-5512.

Joosten, L. A., Heinhuis, B., Abdollahi-Roodsaz, S., Ferwerda, G., Lebourhis, L., Philpott, D. J., Nahori, M. A., Popa, C., Morre, S. A. \& other authors (2008). Differential function of the NACHT-LRR (NLR) members Nod1 and Nod2 in arthritis. Proc Natl Acad Sci U S A 105, 9017-9022.

Kikuchi, T., Matsuguchi, T., Tsuboi, N., Mitani, A., Tanaka, S., Matsuoka, M., Yamamoto, G., Hishikawa, T., Noguchi, T. \& Yoshikai, Y. (2001). Gene expression of osteoclast differentiation factor is induced by lipopolysaccharide in mouse osteoblasts via Toll-like receptors. J Immunol 166, 3574-3579.

Kobayashi, K., Inohara, N., Hernandez, L. D., Galan, J. E., Nunez, G., Janeway, C. A., Medzhitov, R. \& Flavell, R. A. (2002). RICK/Rip2/ CARDIAK mediates signalling for receptors of the innate and adaptive immune systems. Nature 416, 194-199.

Kobayashi, K. S., Chamaillard, M., Ogura, Y., Henegariu, O., Inohara, N., Nuñez, G. \& Flavell, R. A. (2005). Nod2-dependent regulation of innate and adaptive immunity in the intestinal tract. Science 307, 731734 .

Leber, J. H., Crimmins, G. T., Raghavan, S., Meyer-Morse, N. P., Cox, J. S. \& Portnoy, D. A. (2008). Distinct TLR- and NLR-mediated transcriptional responses to an intracellular pathogen. PLoS Pathog 4, e6.

Madrazo, D. R., Tranguch, S. L. \& Marriott, I. (2003). Signaling via Toll-like receptor 5 can initiate inflammatory mediator production by murine osteoblasts. Infect Immun 71, 5418-5421.

Marriott, I. (2004). Osteoblast responses to bacterial pathogens: a previously unappreciated role for bone-forming cells in host defense and disease progression. Immunol Res 30, 291-308.

Marriott, I., Rati, D. M., McCall, S. H. \& Tranguch, S. L. (2005). Induction of Nod1 and Nod2 intracellular pattern recognition receptors in murine osteoblasts following bacterial challenge. Infect Immun 73, 2967-2973.

Marriott, I., Miller, J. R. \& Sahraei, M. (2007). Therapeutic strategies against inflammation and bone loss associated with osteomyelitis. Curr Opin Investig Drugs 8, 887-898.

McCall, S. H., Sahraei, M., Young, A. B., Worley, C. S., Duncan, J. A., Ting, J. P. \& Marriott, I. (2008). Osteoblasts express NLRP3, a nucleotide-binding domain and leucine-rich repeat region containing receptor implicated in bacterially induced cell death. J Bone Miner Res 23, 30-40.

Nemoto, E., Honda, T., Kanaya, S., Takada, H. \& Shimauchi, H. (2008). Expression of functional Toll-like receptors and nucleotidebinding oligomerization domain proteins in murine cementoblasts and their upregulation during cell differentiation. J Periodontal Res 43, 585-593.

Netea, M. G., Ferwerda, G., de Jong, D. J., Jansen, T., Jacobs, L., Kramer, M., Naber, T. H., Drenth, J. P., Girardin, S. E. \& other authors (2005). Nucleotide-binding oligomerization domain-2 modulates specific TLR pathways for the induction of cytokine release. J Immunol 174, 6518-6523.

Ohl, M. E. \& Miller, S. I. (2001). Salmonella: a model for bacterial pathogenesis. Annu Rev Med 52, 259-274.

Reott, M. A., Jr, Ritchie-Miller, S. L., Anguita, J. \& Hudson, M. C. (2008). TRAIL expression is induced in both osteoblasts containing intracellular Staphylococcus aureus and uninfected osteoblasts in infected cultures. FEMS Microbiol Lett 278, 185-192.

Schrum, L. W., Bost, K. L., Hudson, M. C. \& Marriott, I. (2003). Bacterial infection induces expression of functional MHC class II molecules in murine and human osteoblasts. Bone 33, 812-821.

Strober, W., Murray, P. J., Kitani, A. \& Watanabe, T. (2006). Signalling pathways and molecular interactions of NOD1 and NOD2. Nat Rev Immunol 6, 9-20.

Sweet, M. J., Campbell, C. C., Sester, D. P., Xu, D., McDonald, R. C., Stacey, K. J., Hume, D. A. \& Liew, F. Y. (2002). Colony-stimulating factor-1 suppresses responses to CPG DNA and expression of toll-like receptor 9 but enhances responses to lipopolysaccharide in murine macrophages. J Immunol 168, 392-399.

Ting, J. P. \& Davis, B. K. (2005). CATERPILLER: a novel gene family important in immunity, cell death, and diseases. Annu Rev Immunol 23, 387-414.

Yang, S., Takahashi, N., Yamashita, T., Sato, N., Takahashi, M., Mogi, M., Uematsu, T., Kobayashi, Y., Nakamichi, Y. \& other authors (2005). Muramyl dipeptide enhances osteoclast formation induced by lipopolysaccharide, IL-1 alpha, and TNF-alpha through nucleotidebinding oligomerization domain 2-mediated signaling in osteoblasts. J Immunol 175, 1956-1964. 